\title{
Combined assessment of reflow and collateral blood flow by myocardial contrast echocardiography after acute reperfused myocardial infarction
}

F Leclercq, P Messner-Pellenc, Q Descours, J-P Daures, J-L Pasquié, F-X Hager, J-M Davy, R Grolleau-Raoux

\begin{abstract}
Objective-To evaluate the combined assessment of reflow and collateral blood flow by myocardial contrast echocardiography after myocardial infarction.

Design-Myocardial contrast echocardiography was performed in patients with acute myocardial infarction shortly after successful coronary reperfusion (TIMI 3 patency) by direct angioplasty. Collateral flow was assessed before coronary angioplasty, and contrast reflow was evaluated 15 minutes after reperfusion. The presence of contractile reserve was assessed by low dose dobutamine echocardiography (5 to $15 \mu \mathrm{g} / \mathrm{kg} / \mathrm{min}$ ) at (mean (SD)) 3 (2) days after myocardial infarction. Recovery of segmental function (myocardial viability) was evaluated by resting echocardiography at a two month follow up. The study was prospective.
\end{abstract}

Patients-35 consecutive patients referred for acute transmural myocardial infarction.

Results-Contrast reflow was observed in 20 patients $(57 \%)$ and collateral flow in 14 $(40 \%)$. Contrast reflow and collateral contrast flow were both correlated with reversible dysfunction on initial dobutamine echocardiography and at follow up $(p<0.05)$. The presence of reflow or collateral flow on myocardial contrast echocardiography was a highly sensitive $(100 \%)$ but weakly specific $(60 \%)$ indicator of segmental dysfunction recovery. Simultaneous presence of contrast reflow and collateral flow was more specific of reversible dysfunction than reflow alone $(90 \% v$ $60 \%)$.

Conclusions-Combined assessment of reflow and collateral blood flow enhanced the sensitivity of myocardial contrast echocardiography in predicting myocardial viability after acute, reperfused myocardial infarction. The simultaneous presence of reflow and collateral blood flow was highly specific of recovery of segmental dysfunction.

(Heart 1999;82:62-67)

Keywords: contrast echocardiography; coronary reflow; collateral blood flow; dobutamine echocardiography; myocardial dysfunction
After acute reperfused myocardial infarction, myocardial contrast echocardiography appears to be the only available method of assessing intramyocardial reflow, which indicates preservation of microvascular integrity. ${ }^{1-4}$ Ito et al showed that the presence of contrast coronary reflow shortly after reperfusion in acute myocardial infarction was associated with better left ventricular function and less remodelling; conversely, the no reflow phenomenon was a predictor of adverse outcome, particularly with regard to congestive heart failure. ${ }^{5}$ Microvascular integrity can be assessed by myocardial contrast echocardiography after recent myocardial infarction and is an indicator of myocardial reserve-as evaluated by dobutamine echocardiography - and of functional recovery at follow up. ${ }^{6-8}$ However, contrast enhancement shortly after reflow does not necessarily imply regional functional recovery in the chronic stage, because hyperaemia and perfusion-function mismatch result in an overestimate of myocardial salvage with perfusion. ${ }^{7-9}$ Although a strong association was observed by Sabia et al between the presence of contrast collateral blood flow and myocardial viability in patients with recent myocardial infarction, ${ }^{10}$ to our knowledge no study has combined the assessment of reflow and collateral blood flow by myocardial contrast echocardiography shortly after reperfusion of acute myocardial infarction. We therefore designed a study with the following aims: first, to assess the accuracy of combined evaluation of reflow and collateral blood flow using myocardial contrast echocardiography in predicting reversible dysfunction after reperfused myocardial infarction; and second, to compare the predictive value of contrast reflow and contrast collateral blood flow with measurement of contractile reserve by dobutamine echocardiography for evaluating recovery of segmental function at a two month follow up.

\section{Methods}

STUDY POPULATION

Myocardial contrast echocardiography was performed in consecutive patients admitted for their first acute transmural myocardial infarction from January to June 1996. The diagnosis of acute myocardial infarction was made on the basis of chest pain of more than 30 minutes' duration, ST segment elevation of more than 2 $\mathrm{mm}$ in two contiguous ECG leads, and a more than threefold increase in serum creatine 
kinase activity. All patients had echocardiographic images of adequate quality at rest. Informed consent was obtained in each case.

\section{CARDIAC CATHETERISATION}

We only studied patients with a totally occluded infarct related coronary artery at the time of cardiac catheterisation. All patients showed successful coronary reflow on direct angioplasty, defined by TIMI (thrombolysis in myocardial infarction) grade 3 flow and a residual coronary stenosis of less than $30 \%$. According to the TEAM-2 study (second multicenter thrombolytic trials of eminase in acute myocardial infarction), patients who achieved TIMI grade 2 flow were not considered to have myocardial salvage with reperfusion and were therefore not included in the study. ${ }^{11}$ The collateral flow was angiographically graded as absent (no collateral vessels seen) or present (partial or complete filling of the infarct related artery). The right anterior oblique view of the left ventriculogram was used for assessment of global left ventricular function. Left ventricular ejection fraction was calculated from the volumes obtained using the area-length method.

MYOCARDIAL CONTRAST ECHOCARDIOGRAPHY Myocardial contrast echocardiography was performed by manually injecting in the coronary artery a small amount $(2$ to $3 \mathrm{ml}$ ) of sonicated ioxaglate (Hexabrix 320, Guerbet, France) during simultaneously performed transthoracic cross sectional echocardiography in multiple views (mid-papillary short axis view, and apical views of two and four chambers) using a 16 segment model. ${ }^{12} \mathrm{~A}$ Hewlett-Packard Sonos 2000 machine with a $2.5 \mathrm{MHz}$ probe was used (Hewlett-Packard Inc, Andover, Massachusetts, USA).

Echographic images were recorded before and after reperfusion of the culprit coronary artery. Before reperfusion, the microbubble suspension was injected into the contralateral non-infarct-related coronary artery in order to assess the presence of contrast collateral flow, which was considered significant when supplying more than $50 \%$ of the risk area. ${ }^{10}$ The suspension was then injected into the infarct related artery before and 15 minutes after successful reperfusion by direct angioplasty, in order to evaluate the risk and no reflow areas, respectively.

For analysis of contrast images, we used the end diastolic frame of the postinjection cycle showing the best delineation between contrast enhanced and non-enhanced myocardium. ${ }^{3}$ The risk area was determined as the number of segments showing no contrast enhancement before reperfusion. ${ }^{13}$ To minimise the effect of left to left collaterals in patients with occlusion of the left anterior descending coronary artery, we analysed the first end diastolic image after injection of contrast into the left main coronary artery. ${ }^{10}$ No reflow was defined when the residual contrast defect exceeded $25 \%$ of the risk area 15 minutes after angiographic reperfusion. We therefore defined adequate reflow when more than $75 \%$ of the risk area was opacified with contrast. ${ }^{5}$ We refer to this as the "strong" criterion. We also evaluated a weaker criterion of reflow, defined when more than $50 \%$ of the risk area was opacified with contrast ("weak criterion"). ${ }^{36}$

LOW DOSE DOBUTAMINE ECHOCARDIOGRAPHY Dobutamine echocardiography was performed between two and five days after myocardial infarction (average three days) by two experienced operators using a 16 segment left ventricular model. ${ }^{12} \beta$ Blocking agents were withdrawn 24 hours before the test. Dobutamine was infused in a $1 \mathrm{mg} / 1$ solution in three 5 minute periods at 5,10 , and $15 \mu \mathrm{g} / \mathrm{kg} / \mathrm{min}$. In the setting of acute myocardial infarction without limiting coronary stenosis, related to successful angioplasty, high doses of dobutamine were not used. ${ }^{14}$ According to the recommendations of the American Society of Echocardiography, ${ }^{15}$ a semiquantitative score was assigned to each segment: 1 , normokinesia or hyperkinesia; 2, hypokinesia; 3, akinesia; 4, dyskinesia. A wall motion score index was obtained by dividing the sum of the individual segmental scores by the number of segments analysed. Reversible contractile dysfunction was defined as improved wall motion in at least two contiguous dyssynergic segments or in one segment if only two segments were basically dyssynergic. ${ }^{16}{ }^{17} \mathrm{~A}$ change in wall motion from dyskinesia to akinesia or vice versa was considered to indicate unchanged segmental function.

ECHOCARDIOGRAPHIC FOLLOW UP

Follow up resting echocardiography was performed in each patient within two months of hospital discharge (range 28 to 60 days) in order to evaluate recovery of regional dysfunction. All echocardiograms were blindly reviewed on videotape by two independent observers.

\section{STATISTICAL ANALYSIS}

All data are expressed as mean (SD). Sensitivity, specificity, and accuracy were evaluated using standard definitions and expressed in percentages. Fisher's exact test or the $\chi^{2}$ test was performed to compare categorical variables between different groups of patients in relation to the prediction of reversible dysfunction at follow up. For comparison between continuous variables, a non-parametric

Table 1 Clinical and angiographic characteristics of the study patients

\begin{tabular}{ll}
\hline Number of patients & 35 \\
Mean (range) age (years) & $59(24$ to 78$)$ \\
Male & $27(77)$ \\
Diabetes mellitus & $7(20)$ \\
Hypertension & $9(26)$ \\
Anterior infarction & $20(57)$ \\
Mean (range) LVEF (\%) & $52(20$ to 70$)$ \\
LVEF $\leqslant 35 \%$ & $8(23)$ \\
Infarct related artery & $19(54)$ \\
$\quad$ Left anterior descending & $1(3)$ \\
Left main & $15(43)$ \\
$\quad$ Right coronary artery & $17(48)$ \\
Multivessel disease & $11(31)$ \\
Angiographic collateral blood flow & \\
\hline
\end{tabular}

Values are $\mathrm{n}(\%)$ unless otherwise stared. LVEF, left ventricular ejection fraction. 
Table 2 Clinical and angiographic characteristics of patients studied by myocardial contrast echocardiography: reflow and collateral blood flow assessment

\begin{tabular}{lllll}
\hline & Reflow & No reflow & Collateral flow (+) & Collateral flow (-) \\
\hline Number of patients & 20 & 15 & 14 & 21 \\
Mean (range) age (years) & $63(24$ to 76$)$ & $56(44$ to 78$)$ & $51(32$ to 76$)$ & $56(24$ to 78$)$ \\
Hypertension & $5(25)$ & $4(26)$ & $4(28)$ & $5(24)$ \\
Diabetes mellitus & $4(20)$ & $3(20)$ & $3(21)$ & $4(19)$ \\
Anterior infarct location & $11(55)$ & $9(60)$ & $8(57)$ & $12(57)$ \\
Multivessel disease & $8(40)$ & $9(60)$ & $7(50)$ & $7(33)$ \\
Angiographic collateral flow & $6(30)$ & $5(33)$ & $4(28)$ & $66(20$ to 73$)$ \\
Mean (range) LVEF (\%) & $61^{\star}(50$ to 73$)$ & $43^{\star}(20$ to 65$)$ & $55(39$ to 73$)$ & 340 \\
Mean delay of reperfusion (min) & 230 & 310 & 270 & \\
\hline
\end{tabular}

Values are $\mathrm{n}(\%)$ unless otherwise stated.

$\star_{\mathrm{p}}<0.05$.

LVEF, left ventricular ejection fraction.

Kruskal-Wallis test was used. Differences between comparisons were considered significant at $\mathrm{p}$ value of $<0.05$.

\section{Results}

PATIENT CHARACTERISTICS

Among the 38 patients eligible for the study, one died during angioplasty from cardiogenic shock and two were lost to follow up after hospital discharge. The clinical and angiographic characteristics of the 35 patients included are summarised in table 1 .

\section{MYOCARDIAL CONTRAST ECHOCARDIOGRAPHY} Contrast coronary reflow

Twenty patients (57\%) had more than $75 \%$ of the risk area uniformly opacified with contrast after angiographic reperfusion of the infarct related coronary artery (contrast reflow), whereas 15 patients $(43 \%)$ had a residual contrast defect of more than $25 \%$ (contrast no reflow). A residual contrast defect of more than $50 \%$ of the risk area was observed in six patients $(17 \%)$. Table 2 summarises the characteristics of these two groups. Only a greater left ventricular ejection fraction was statistically associated with the presence of a contrast reflow $(\mathrm{p}<0.05)$.

Contrast collateral blood flow

Significant contrast collateral blood flow was observed before reperfusion in 14 patients $(40 \%)$ and was not statistically associated with the presence of angiographically confirmed collateral blood flow $(p=0.39)$. Clinical and angiographic characteristics did not differ significantly between patients with and without the presence of contrast collateral flow (table 2).

DOBUTAMINE AND FOLLOW UP

ECHOCARDIOGRAPHY

Functional recovery with dobutamine was more often observed in patients with reflow

Table 3 Contrast echocardiography results regarding recovery of segmental function at two month follow up

\begin{tabular}{lll}
\hline & \multicolumn{2}{l}{ Two month follow up } \\
\cline { 2 - 3 } Contrast echocardiography & $\begin{array}{l}\text { Improved } \\
\text { contraction }\end{array}$ & $\begin{array}{l}\text { Unchanged } \\
\text { contraction }\end{array}$ \\
\hline Reflow $(\mathrm{n}=20,57 \%)$ & $14(70)$ & $6(30)$ \\
No reflow $(\mathrm{n}=15,43 \%)$ & $4(27)$ & $11(73)$ \\
Collateral flow $(-)(\mathrm{n}=21,60 \%)$ & $7(33)$ & $14(67)$ \\
Reflow and/or collateral $(+)(\mathrm{n}=21,60 \%)$ & $15(71)$ & $6(29)$ \\
Reflow and collateral $(+)(\mathrm{n}=8,23 \%)$ & $6(75)$ & $2(25)$ \\
No reflow and collateral $(-)(\mathrm{n}=6,17 \%)$ & 0 & $6(100)$ \\
\hline
\end{tabular}

Values are $\mathrm{n}(\%)$. than in those without reflow $(p=0.04)$ and was statistically associated with recovery of contractile function at follow up $(\mathrm{p}=0.03)$.

Mean wall motion score index with dobutamine (1.32 $v 1.44$ at baseline; $\mathrm{p}<0.05)$ and at follow up (1.26; p $<0.05 v$ baseline) improved in the patients with contrast collateral blood flow, whereas no significant change was observed in the patients without contrast collateral flow. The presence of contrast collateral blood flow was also statistically associated with improvement of segmental function with dobutamine $(p=0.04)$ and at follow up $(\mathrm{p}=0.04)$.

PREDICTION OF REVERSIBLE DYSFUNCTION AT TWO MONTH FOLLOW UP

Myocardial contrast echocardiography

Contrast reflow and contrast collateral blood flow evaluated alone or in combination were both associated with recovery of function at follow up ( $<<0.05$; table 3 ). Figure 1 is an example of a patient with anterior myocardial infarction related to left anterior descending coronary artery occlusion and with collateral flow originated from the right coronary artery. This patient had reversible contractile dysfunction at two month follow up echocardiography. Table 4 summarises the sensitivity, specificity, and negative and positive predictive values of myocardial contrast echocardiography - with and without combining the assessment of reflow and collateral blood flow-in predicting recovery of contractile function at two months. Presence of either reflow ("strong" criterion) or collateral blood flow was highly sensitive $(100 \%)$ of recovery of segmental function at follow up. The use of the weak reflow criterion (>50\% of the risk area reperfused with contrast) was very sensitive $(100 \%)$ but poorly specific $(30 \%)$ of contractile reserve at follow up.

Myocardial contrast $v$ dobutamine echocardiography

Myocardial contrast echocardiography had a lower specificity than dobutamine echocardiography with respect to the presence of myocardial viability at follow up (60\% v 90\%; table 4$)$. Only the simultaneous presence of contrast reflow and contrast collateral blood flow resulted in similar values of specificity for the two tests $(90 \%)$ without decreasing sensitivity. Dobutamine echocardiography remained less sensitive than myocardial contrast echocardiography (60\% v 100\%). 

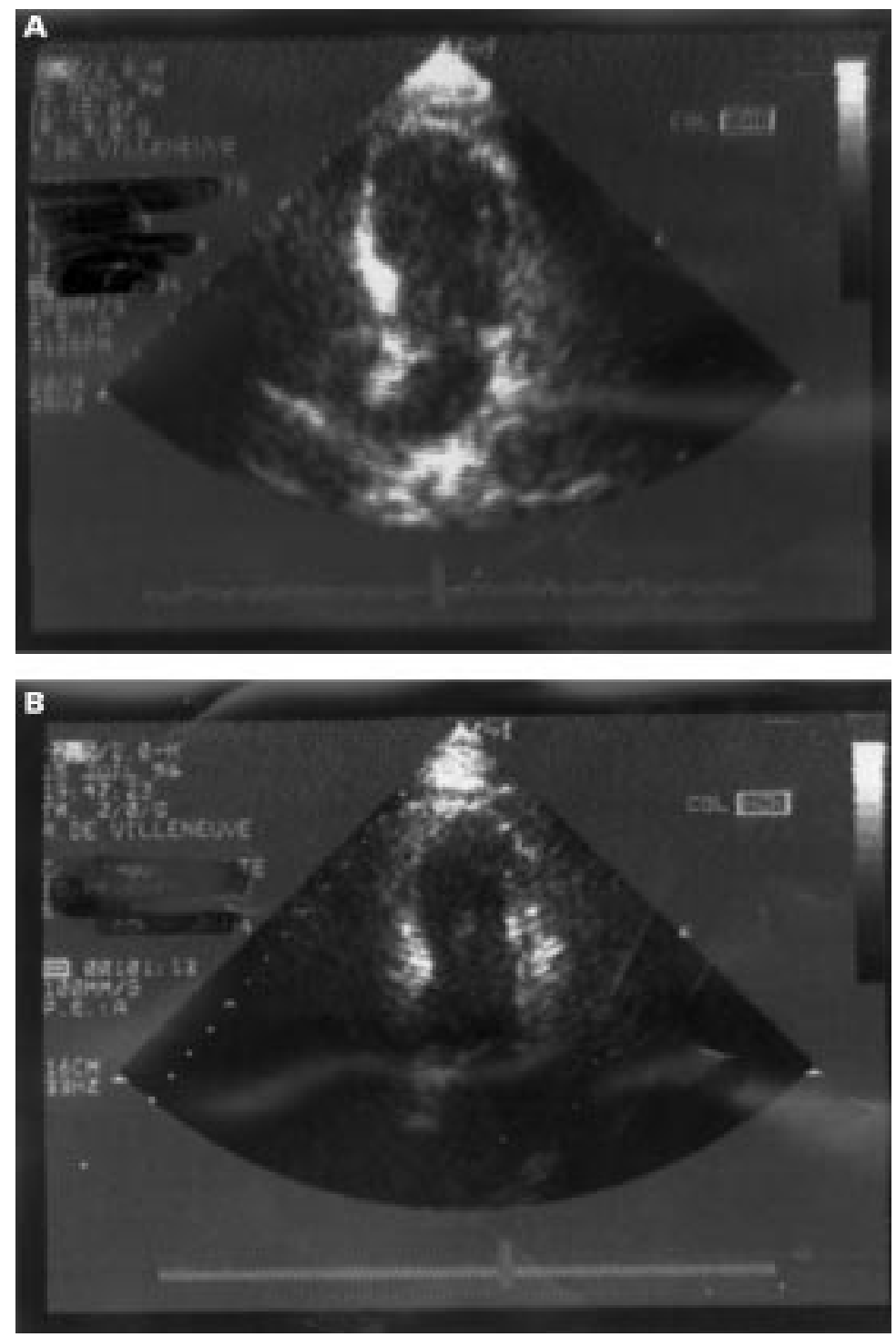

Figure 1 Apical view of two chambers of the left ventricle obtained in a patient with acute anterior myocardial infarction linked to a left anterior descending (LAD) coronary artery occlusion. In the upper panel, sonicated ioxaglate was injected in the left main coronary artery before angioplasty: the injection defined the perfusion bed supplied by the LAD (risk area): apical, mid, and basal anterior segments of the left ventricle were not opacified by the contrast. In the lower panel, sonicated ioxaglate was injected in the right coronary artery. Homogeneous opacification of the basal and mid-inferior segments was observed (perfusion bed of the right coronary artery). Simultaneous opacification of the basal and mid-anterior segments of the left ventricle was linked to collateral blood flow originated from the right coronary system when the LAD was occluded.

Table 4 Diagnostic accuracy of dobutamine echocardiography and myocardial contrast echocardiography for prediction of improvement of contractile function at two month follow up

\begin{tabular}{lcllc}
\hline & $\begin{array}{l}\text { Sensitivity } \\
(\%)\end{array}$ & $\begin{array}{l}\text { Specificity } \\
(\%)\end{array}$ & PPV (\%) & NPV (\%) \\
\hline Dobutamine echocardiography & 60 & 90 & 86 & 69 \\
Contrast reflow 75\% & 70 & 60 & 64 & 67 \\
Contrast reflow 50\% & 100 & 30 & 58 & 100 \\
Contrast collateral flow (+) & 60 & 70 & 64 & 63 \\
Contrast reflow 75\% and/or collateral flow & 100 & 60 & 69 & 100 \\
Contrast reflow 75\% and collateral flow & 45 & 90 & 75 & 62 \\
\hline
\end{tabular}

Reflow $75 \%, 75 \%$ of the risk area reperfused with contrast ("strong criterion”).

Reflow $50 \%, 50 \%$ of the risk area reperfused with contrast ("weak criterion").

PPV, positive predictive value; NPV, negative predictive value.

Other variables associated with myocardial viability

Lower mean age (51 v 59 years; $\mathrm{p}=0.02)$, absence of previous angina $(\mathrm{p}=0.007)$, and lower mean wall motion score index at two months (1.26 (0.18) v $1.45(0.23) ; \mathrm{p}=0.04)$ were the only clinical variables statistically associated with presence of myocardial viability at the two month follow up.

\section{Discussion}

The main results of our study were first, that myocardial reflow and collateral blood flow, evaluated by myocardial contrast echocardiography shortly after reperfusion of acute myocardial infarction, were both statistically associated with recovery of contractile function at a two month follow up; second, that the combined assessment of these two variables is highly sensitive $(100 \%)$ for myocardial viability, without decreasing the specificity of the test; and third, that dobutamine echocardiography remains more specific than contrast echocardiography in predicting myocardial viability $(90 \%$ v 60\%). Only the simultaneous presence of reflow and collateral blood flow using contrast echocardiography achieved values of specificity similar to that of dobutamine echocardiography.

MYOCARDIAL CONTRAST NO REFLOW AFTER ACUTE MYOCARDIAL INFARCTION

The air filled bubbles used for myocardial contrast echocardiography have intravascular rheology similar to that of red cells ("microvascular tracer") and can be used safely in the acute phase of myocardial infarction to evaluate the presence of intramyocardial reflow, a fundamental prerequisite for myocardial viability. ${ }^{138} 8$ 18-21 Although hyperaemia occurring in the early phase of reflow leads classically to an underestimation of irreversibly damaged microvasculature, ${ }^{7} 81822{ }^{23}$ Villanueva et al showed that the contrast defect size (no reflow area) correlated with infarct size 15 minutes after reperfusion of experimental myocardial infarction. ${ }^{24}$ Ito et al, who performed myocardial contrast echocardiography before and shortly after coronary reperfusion, also observed a good correlation between contrast reflow and patient prognosis, particularly with regard to congestive heart failure..$^{5}$ In our study, the no reflow phenomenon was observed in 15 patients (43\%), more commonly than reported recently by Ito et al (37\%), and much more commonly than observed by Illiceto et al (25\%) or Ito et al in their original publication (25\%), where no reflow was defined as a residual contrast defect involving more than $50 \%$ of the risk area after reperfusion. ${ }^{325}$ Using this latter definition, however, this phenomenon was observed in six (17\%) of our patients. As did other investigators, we observed that no reflow was statistically more common in larger infarcts (lower mean ejection fraction and higher mean wall motion score index) and that, conversely, the presence of reflow was statistically associated with both contractile reserve by dobutamine echocardiography and recovery of regional function at follow up. 357182627

COMBINED ASSESSMENT OF REFLOW AND COLLATERAL BLOOD FLOW BY MYOCARDIAL CONTRAST ECHOCARDIOGRAPHY

Because the size of the bubbles of the sonicated agents (7 to $10 \mu \mathrm{m}$ ) is small enough to resolve 
most collateral vessels, myocardial contrast echocardiography may be the most suitable technique for the assessment of collateral flow. ${ }^{20-31}$ Even though the development of collateral channels usually requires several hours after coronary occlusion, beneficial effects of angiographically identified collateral vessels on left ventricular function have been reported within two to eight hours after the onset of symptoms. ${ }^{32}{ }^{33}$ Thus significant contrast collateral flow was observed in 14 of our patients $(40 \%)$ and, as in the results of Sabia et $a l,{ }^{10}$ this finding was associated with reversible dysfunction at follow up but did not correlate with the collateral vessel grade determined angiographically $(\mathrm{p}=0.04)$. However, in our study - and reported for the first time to our knowledge - the combined assessment of reflow and collateral blood flow after acute reperfused myocardial infarction enhanced the sensitivity of myocardial contrast echocardiography for detecting reversible dysfunction, without decreasing the specificity of the test $(100 \%$ sensitivity in presence of either reflow or collateral blood flow, $v 70 \%$ with reflow alone and $60 \%$ with collateral flow alone; table 4). The sensitivity of reflow alone in our study $(70 \%)$ was lower than the results observed by Illiceto et al or Agati et al (100\%), but this difference is probably related to different definitions of reflow in these studies. ${ }^{25}{ }^{34}$ In our study, the choice of the strong criterion to define reflow (more than $75 \%$ of the risk area opacified by contrast) decreased the sensitivity but enhanced the specificity of the test $(60 \%$, v $46 \%$ observed by Illiceto et al). The combined assessment of reflow and collateral blood flow resulted in high sensitivity of myocardial contrast echocardiography for myocardial viability, whereas the choice of the strong criterion to define reflow resulted in better specificity (table 4). Thus the combined evaluation of reflow and collateral flow enhanced the accuracy of myocardial contrast echocardiography in predicting functional recovery, which seems to be particularly relevant in patients with depressed left ventricular function. ${ }^{35}$ Since residual blood flow would maintain myocardial cell viability for a prolonged period, our results confirm that collateral flow within the occluded bed may be an important factor in determining infarct size in humans. ${ }^{27} 283132$ By increasing the accuracy of the test, the combined assessment of reflow and collateral blood flow using contrast echocardiography may improve the identification of high risk patients, who are most susceptible to developing ventricular remodelling and heart failure. ${ }^{5}$ Further studies are needed, however, to define the role of early vasodilator treatment in these patients.

CONTRACTILE RESERVE $v$ MICROVASCULAR INTEGRITY IN PREDICTING RECOVERY OF SEGMENTAL DYSFUNCTION

Perfusion may overestimate the amount of myocardial salvage, particularly during the early hours after reperfusion, and thus myocardial contrast echocardiography is less specific than dobutamine echocardiography in predicting myocardial viability. ${ }^{75343637}$ Bolognese et al recently showed that contrast enhancement shortly after reflow did not necessary imply functional recovery in the chronic stage. ${ }^{7}$ They also showed that contractile reserve elicited by dobutamine echocardiography was more accurate than early reperfusion as assessed by myocardial contrast echocardiography in predicting functional recovery in patients reperfused by direct angioplasty. One explanation for these findings is that areas that are defined as non-viable by dobutamine echocardiography and have reflow with contrast echocardiography are areas with metabolism but no function. ${ }^{9}$ Our results are quite similar when the no reflow phenomenon was considered on its own. However, combined evaluation of reflow and collateral blood flow allows a high sensitivity $(100 \%)$ and negative predictive value $(100 \%)$ to be achieved, with a specificity $(60 \%)$ and positive predictive value (64\%) better than those obtained by Bolognese et al (18\% and $41 \%$, respectively). Even though combined assessment of reflow and collateral flow by contrast echocardiography still yields suboptimal information for predicting functional recovery, evaluation of these two variables enhanced the accuracy of the test compared with evaluating reflow alone (table 4). Because the extent of collateral flow within the occluded bed was one of the main determinants of final infarct size, ${ }^{10} 29$ the combined assessment of reflow and collateral flow was probably more representative of "myocardium at risk" and thus explain our results. The difference in the results observed between contrast and dobutamine echocardiography may partly be explained by differences in the timing of the tests. The relatively low sensitivity of dobutamine echocardiography in our study compared with previous results was probably linked to inclusion of inferoposterior myocardial infarcts ( $43 \%$ of patients) and to the use of harder criteria of myocardial viability with dobutamine echocardiography (improvement of wall motion in at least two contiguous dyssynergic segments) which enhances the specificity but may decrease the sensitivity of the test. ${ }^{74}$

\section{STUDY LIMITATIONS}

Coronary angiography was not performed systematically at follow up and therefore reocclusion or restenosis - which may alter functional recovery - cannot definitely be excluded. Contrast collateral blood flow was evaluated qualitatively (that is, present or absent) and not as a percentage of the infarct bed perfused by the non-infarct-related coronary artery. ${ }^{10}{ }^{29}$ However, Sabia et al observed that only patients in whom more than $50 \%$ of the infarct bed was supplied by collateral flow had improved function at follow up. ${ }^{10}$ We therefore chose this definition of "significant collateral blood flow." For clinical routine, particularly in patients treated with thrombolysis, the intravenous administration of contrast may be a very promising way of evaluating perfusion non-invasively. ${ }^{38} 39$ In the setting of an acute myocardial infarct reperfused by direct angioplasty, the intracoronary injection of contrast in the guiding catheter 
after reperfusion may enable prompt assessment, in the cardiac catheterisation laboratory, of both coronary anatomy and the quality of microvascular perfusion. However, intravenous agents for evaluating perfusion are not yet approved in France.

CONCLUSIONS

Combined assessment of reflow and collateral blood flow shortly after reperfusion of acute myocardial infarction enhanced the sensitivity of myocardial contrast echocardiography for identifying the presence of reversible contractile dysfunction. The simultaneous presence of reflow and collateral blood flow is as specific as contractile reserve evaluated by dobutamine echocardiography. This direct, rapid, and safe assessment of myocardial viability using myocardial contrast echocardiography may be a help in the evaluation of prognosis immediately after angiographic reperfusion.

1 Kloner RA, Ganote CE, Jennings B. The no reflow phenomenon after temporary coronary occlusion in the phenomenon after temporary coronar
dog. F Clin Invest 1974;54:1496-508

2 Krug A, Du Mesnil de Rochement W, Korb G. Blood supply to the myocardium after temporary coronary occlusion in the dog. Circ Res 1966;19:57-62.

3 Ito H, Tomooka T, Sakai N, et al. Lack of myocardial perfusion immediately after successful thrombolysis. A predictor of poor recovery of left ventricular function in anterior myocardial infarction. Circulation 1992;85:1699705.

4 Illiceto S, Marangelli V, Marchese A, et al. Myocardial contrast echocardiography in acute myocardial infarction. Pathophysiological background and clinical applications. Eur Heart F 1996; 17:344-53.

5 Ito $\mathrm{H}$, Maruyama A, Iwakura $\mathrm{K}$, et al. Clinical implications of the no reflow phenomenon. A predictor of complications and left ventricular remodeling in reperfused anterior wall myocardial infarction. Circulation 1996;93:223-8.

6 Illiceto S, Galiuto L, Marchese A, et al. Functional role of microvascular integrity in patients with infarct-related pat-
ency after acute myocardial infarction. Eur Heart $f$ ency after acute

7 Bolognese L, Antoniucci D, Rovai D, et al. Myocardial contrast echocardiography versus dobutamine echocardiography for predicting functional recovery after acute myocardial infarction treated with primary coronary angioplasty. F Am Coll Cardiol 1996;28:1677-83.

8 Ito $\mathrm{H}$, Iwakura $\mathrm{K}, \mathrm{Oh} \mathrm{H}$, et al. Temporal changes in myocardial perfusion patterns in patients with reperfused anterior wall myocardial infarction. Their relation to myocardial viability. Circulation 1995;91:656-62.

9 Bax JJ, Valkema R, Visser FC, et al. FDG SPECT in the assessment of myocardial viability. Comparison with dobutamine echocardiography. Eur Heart F 1997;18(suppl D):D124-9.

10 Sabia PJ, Powers ER, Ragosta M, et al. An association between collateral blood flow and myocardial viability in patients with recent myocardial infarction. $N$ Engl $f \mathrm{Med}$ patients with recent

11 Karagounis C, Sorensen SG, Menlove RL, et al, for the TEAM-2 Investigators. $\mathcal{F}$ Am Coll Cardiol 1992;19:1-10.

12 Picano E, Mathias W, Pingitore A, et al, on behalf of the Echo Dobutamine International Cooperative Study Group. Safety and tolerability of dobutamine-atropine stress echocardiography: a prospective multicenter study. Lancet 1994;344:1190-2.

13 Kaul S, Glasheen W, Ruddy TD, et al. The importance of defining left ventricular area at risk in vivo during acute myocardial infarction: an experimental evaluation with myocardial contrast two-dimensional echocardiography. Circulation 1987;75:1249-60.

14 Kaul S. Dobutamine echocardiography for determining myocardial viability after reperfusion: experimental and
clinical observations. Eur Heart $\mathcal{F}$ 1995;16(suppl M):1723.

15 Schiller NB, Shah PM, Crawford M, et al. Recommendations for quantification of the left ventricle by twotions for quantification of the left ventricle by two-
dimensional echocardiography. $f \mathrm{Am}$ Soc Echocardiogr 1989;2:358-67.

16 Smart SC, Sawada S, Ryan T, et al. Low-dose dobutamine echocardiography detects reversible dysfunction after thrombolytic therapy of acute myocardial infarction. Circulation 1993;88:405-15.
17 Leclercq F, Messner-Pellenc P, Moragues C, et al. Myocardial viability assessed by dobutamine echocardiography in acute myocardial infarction after successful primary coronary angioplasty. Am $\mathcal{f}$ Cardiol 1997;80:6-10.

18 Ragosta M, Camarano G, Kaul S, et al. Microvascular integrity indicates myocellular viability in patients with recent myocardial infarction. New insights using myocardial contrast echocardiography. Circulation 1994;89:2562-

19 Kaul S. Clinical applications of myocardial contrast echocardiography. Am f Cardiol 1992;69:46-55H

20 Feinstein S, Lang R, Neumann A. Contrast echocardiography during coronary arteriography in humans: perfusion and anatomic studies. F Am Coll Cardiol 1988;11:5965.

21 Lang R, Borow KM, Neumann A, et al. Effect of intracoronary injections of sonicated microbubbles on left ventricuar contractility. Am 7 Cardiol 1987;60:166-71.

22 Jeremy RW, Links JM, Becker LC. Progressive failure of coronary flow during reperfusion of myocardial infarction: documentation of the no reflow phenomenon with positron emission tomography. 7 Am Coll Cardiol 1990;16:695-704.

23 White FC, Sanders M, Bloor CM. Regional redistribution of myocardial blood flow after coronary occlusion and reperfusion in the conscious dog. Am $\mathcal{F}$ Cardiol 1978;42: $234-43$

24 Villanueva FS, Glasheen WP, Sklenar J, et al. Characterization of spatial patterns of flow within the reperfused myocardium by myocardial contrast echocardiography. Implications in determining extent of myocardial salvage. Circulation 1993;88:2596-606.

25 Illiceto S, Galiuto L, Marchese A, et al. Analysis of microvascular integrity, contractile reserve, and myocardial viability after acute myocardial infarction by dobutamine echocardiography and myocardial contrast echocardiography. Am f Cardiol 1996;77:441-5.

26 Camarano G, Ragosta M, Gimple LW, et al. Identification of viable myocardium with contrast echocardiography in patients with poor left ventricular function caused by recent or remote myocardial infarction. Am f Cardiol 1995; 75:215-19.

27 Kenner M, Zajac EJ, Kondos GT, et al. Ability of the no-reflow phenomenon during an acute myocardial infarction to predict left ventricular dysfunction at one-month follow up. Am F Cardiol 1995;76:861-8.

28 Cohen M, Rentrop KP. Limitation of myocardial ischemia by collateral circulation during sudden controlled coronary artery occlusion in human subjects: a prospective study. Circulation 1986;74:469-76.

29 Sabia PJ, Powers ER, Jayaweera AR, et al. Functional significance of collateral blood flow in patients with recent acute myocardial infarction. A study using myocardial contrast echocardiography. Circulation 1992;85:2080-9.

30 Widimsky P, Cornel JH, Ten Cate FJ. Evaluation of collateral blood flow by myocardial contrast echocardiography. Br Heart $\mathcal{F}$ 1988;59:20-2.

31 Lim Y, Nanto S, Masuyama T, et al. Coronary collaterals assessed with myocardial contrast echocardiography in healed myocardial infarction. Am 7 Cardiol 1990;66:55661 .

32 Saito $\mathrm{Y}$, Yasuno M, Ishida M, et al. Importance of coronary collaterals for restoration of left ventricular function after intracoronary thrombolysis. Am f Cardiol 1985;55:125963.

33 Rentrop KP, Feit F, Sherman W, et al. Late thrombolytic therapy preserves left ventricular function in patients with collateralized total coronary occlusion: primary end points findings of the second Mount Sinai-New York University reperfusion trial. 7 Am Coll Cardiol 1989;14:58-64.

34 Agati L, Voci P, Autore C, et al. Combined use of dobutamine echocardiography and myocardial contrast echocardiography in predicting regional dysfunction recovery after coronary revascularization in patients with recent myocardial infarction. Eur Heart f 1997;18:771-9.

35 Marwick TH. The viable myocardium: epidemiology, detection, and clinical implications. Lancet 1998;351:81519.

36 De Filippi CR, Willett DL, Irani WN, et al. Comparison of myocardial contrast echocardiography and low-dose dobutamine echocardiography in predicting recovery of left ventricular function after coronary revascularization in chronic ischemic heart disease. Circulation 1995;92:28638.

37 Rovai D, Zanchi M, Lombardi M, et al. Residual myocardial perfusion in reversibly damaged myocardium by dipyridamole contrast echocardiography. Eur Heart f 1996;17:296301.

38 Porter TR, Li S, Kilzer $\mathrm{K}$, et al. Effect of significant two-vessel versus one-vessel coronary artery stenosis on
myocardial contrast defects observed with intermittent harmonic imaging after intravenous contrast injection during dobutamine stress echocardiography. $7 \mathrm{Am}$ Coll Cardiol 1997;30:1399-406.

39 Kaul S, Senior R, Dittrich H, et al. Detection of coronary artery disease with myocardial contrast echocardiography. sion computed tomography. Circulation 1997;96:785-92. 